\title{
Towards Clinical Application of Patients' Radiosensitivity Data
}

H. Thierens ${ }^{\mathrm{a}}$, K. De Ruyck ${ }^{\mathrm{b}}$, J. Werbrouck ${ }^{\mathrm{a}}$, P. Willems ${ }^{\mathrm{a}}$, A. Vral ${ }^{\mathrm{a}}$, F. Duprez ${ }^{\mathrm{c}}$, M. Van Eijkeren ${ }^{\mathrm{c}}$ and W. De Neve ${ }^{\mathrm{c}}$

${ }^{a}$ Dept Basic Medical Sciences, University Hospital Ghent, Building B3, De Pintelaan 185, 9000 Gent, Belgium; ${ }^{\text {b } G h e n t ~ U n i v e r s i t y, ~ P r o e f t u i n s t r a a t ~ 86, ~} 9000$ Gent, Belgium; ${ }^{\mathrm{c}}$ Radiotherapy Dept, University Hospital Ghent, Building P\%, De Pintelaan 185, 9000 Gent, Belgium

hubert.thierens@ugent.be

INTRODUCTION The ultimate goal of curative radiotherapy is to achieve tumor control without producing complications due to damage to surrounding normal tissues. Radiotherapy dose fractionation schemes are based on $\alpha / \beta$ values and the TD5/5 tolerance dose producing $5 \%$ of a specific radiotoxic effect within 5 years. Normal tissue radiation effects are due to a large extent to interindividual differences in response between patients, which is determined mainly by genetic factors. The first indications for the inherited basis for clinical radiosensitivity came from patients with rare genetic syndromes, associated with germline mutations in genes involved in the detection and repair of DNA damage (1). In addition, very radiosensitive patients without these genetic syndromes present often mutations in DNA repair genes and genes related to cytokines as TGF $\beta 1$. The importance of the individual patient radiosensitivity assessment has increased during the last years in the application of more recent radiotherapy modalities as intensity modulated radiotherapy (IMRT). These techniques easily allow dose escalation and de-escalation studies for patients. Data available today indicate that clinical normal tissue radiosensitivity should be considered as a complex phenotype dependent on the interplay of several gene products: genetic determinants exhibit differential expression in different cell types and tissues (2). Single nucleotide polymorphisms (SNPs) represent approximately 90\% of the naturally occurring sequence variation in the human genome. So common SNPs in a number of genes related to the considered radiopathologic effects could be the inherited basis of clinical radiosensitivity. In the past decade different in vitro radiosensitivity assays have been developed aimed at prediction of individual radiosensitivity and individualizing treatment schedules. Application of the colony-forming assay of primary skin fibroblasts, obtained from a skin biopsy of patients was rather disappointing in the assessment of the individual clinical radiosensitivity and is not adapted to be used in routine clinical practice. With respect to tests evaluating DNA damage and repair, initially encouraging results were obtained with the alkaline comet assay (3). However, larger scale studies later on did not support the value of the comet assay for use in clinical practice. At the moment a number of research groups investigate the scoring of $\gamma$-H2AX foci after in vitro irradiation as a marker of individual radiosensitivity. Among the cytogenetic test systems on lymphocytes, the G2 chromatid break assay seems to be the most representative for chromosomal radiosensitivity assessment. Patients with a high clinical radiosensitivity had a significantly higher G2 score compared to normal reactors (4). The usefulness of the in vitro micronucleus assay for predicting normal tis- 
sue response is controversial. G2 ASSAY In the G2 assay, chromatid breaks are scored in metaphases of cultured lymphocytes, irradiated in vitro 90 min before harvesting. The number of chromatid breaks per metaphase, the G2 score, is a measure for chromosomal radiosensitivity. Different research groups have shown the association between chromosomal radiosensitivity and genetic predisposition to different types of cancer, especially breast cancer and head and neck cancer $(5,6)$. The relation between chromosomal and clinical radiosensitivity is less clear. Our research group performed a large scale study on chromosomal radiosensitivity and late radiation-induced damage after radiotherapy in 62 patients treated for gynecological tumors (7). A clear correlation between the G2 data and the clinical response scored according to the CTC scale at the population level was found. A similar recent study of acute reactions in head and neck cancer patients treated with IMRT by our research group was less conclusive: sorting patients out in a group with low and moderate radiosensitivity (CTC0-2) and a group with high radiosensitivity $(\mathrm{CTC} 3+)$ resulted in no statistical significant difference between the G2 scores for dermatitis, mucositis and dysphagia. In conclusion, where the relation between chromosomal radiosensitivity and cancer predisposition at the population group level is well established, the G2 score cannot be used as a clinical screen for individual clinical radiosensitivity. RADIOGENOMICS Clinical normal tissue radiosensitivity should be regarded as a complex trait dependent on the interplay of several gene products. Individual variations in expression of these genes are responsible for variations in clinical radiosensitivity in different cell types and tissues. SNPs and other small germline mutations in these genes lie at the basis of this differential expression. With respect to clinical radiosensitivity, two groups of genes are considered for SNP analysis in radiogenomics studies: genes responsible for recognition and repair of DNA damage and genes related to cytokines and growth factors. The first group is more related to acute radiotoxicity, while the second group is more linked to late effects. The ATM gene encodes a kinase that initiates processes that regulate DNA repair, cell cycle checkpoints and apoptosis. With respect to DNA repair, genes involved in base excision repair (e.g. XRCC1, APE1, OGG1, PARP), homologous recombination (e.g. XRCC3, $\operatorname{Rad} 50, \operatorname{Rad} 51$ ) and non-homologous end-joining (e.g. Ku70, Ku80, Lig4) are considered for SNP analysis. Candidates among cytokines for SNP analysis related to tissue response to ionising radiation are e.g. TNF- $\alpha$, IL1, IL6, IL8, IL10, TGF $\beta 1$, PDGF. In view of the central role of TGF $\beta 1$ in the development of radiation induced tissue reactions, genes coding for the most important TGF $\beta 1$ signal transduction proteines are also considered: TGF $\beta$ R1, TGF $\beta$ R2, Smad2, Smad3. We performed already different radiogenomic studies for the association between the genotypic profile of patients and the severity of complications of radiotherapy. In the gynaecological cancer patient population considered for the G2 study, we also investigated the association between the occurrence of late moderate (CTC2) and severe complications (CTC3+) and the presence of the Arg194Trp, Arg280His, Arg399Gln, Gln632Gln SNPs in XRCC1, the 5'UTR 4.541A $>$ G, IVS5-14 A>G, Thr241Met SNPs in XRCC3 and the Ser326Cys SNP in OGG1 (7). Furthermore, the impact of the -1552 delAGG, $-800 \mathrm{G}>\mathrm{A},-509 \mathrm{C}>\mathrm{T}$, Leu10Pro, Arg25Pro, Thr263IIe 
polymorphisms in TGF $\beta 1$ was studied for an expanded population (8). With respect to polymorphisms in DNA repair genes, the XRCC1 (Arg280His, Arg399Gln, Gln632Gln) and the XRCC3 (IVS5-14 A $>$ G) variant alleles were found to be risk alleles $(\mathrm{OR}>1)$ for moderate and severe late complications (CTC2+). Presence of three or more of these alleles results in an OR of 10 and more $(p=0.001)$. With respect to TGF $\beta 1$, the homozygous variant -1552 delAGG, $-509 \mathrm{TT}$ and 10 Pro genotypes in TGF $\beta 1$ were found to be associated with the risk of developing late severe $(\mathrm{CTC} 3+)$ radiotherapy reactions and triple variant homozygous patients had a 3.6 times increased risk. Recently, a radiogenomics study of acute reactions (dermatitis, mucositis, dysphagia) in 88 head and neck cancer patients treated with IMRT was finished focussing on SNPs in the DNA DSB repair genes XRCC3 (5'UTR 4.541A > G, IVS5-14 A > G, Thr241Met), Rad51 (5'UTR 135G >C, 5'UTR 172G $>$ T), Lig4 (Thr9Ile, Asp568Asp), Ku70 (5'UTR 61C>G) and Ku80 (IVS21$2.804 \mathrm{G}>\mathrm{A}$ ). The XRCC3 codon 241 and the Ku70 5'UTR variant genotypes are significantly $(\mathrm{p}<0.05)$ associated with the risk of developing severe dysphagia $(\mathrm{CTC} 3+)$. Analysis of the data also highlighted the importance of physical dose to the critical structures, which is strongly dependent on the individual treatment in recent radiotherapy techniques as IMRT. Based on the genotypic data and dose volume histograms of the constrictor pharyngeus muscles, a risk model for severe dysphagia was developed with a sensitivity of $77 \%$ and an specificity of $78 \%$. This study will be expanded with the inclusion of more patients and a panel of SNPs in genes involved in DNA repair and cytokines representing risk alleles according to our own work and literature data. Also a clinical follow up for late complications is started. This will lead to optimization of the prediction model, which can then be applied for patient selection in dose escalation and de-escalation studies. REFERENCES (1)Gatti RA. The inherited basis of human radiosensitivity. Acta Oncol 2001; 40:702-711. (2)Russell NS, Begg AC. Editorial radiotherapy and oncology 2002: Predictive assays for normal tissue damage. Radiother Oncol 2002; 64:125129. (3)Alapetite $\mathrm{C}$ et al. Analysis by alkaline comet assay of cancer patients with severe reactions to radiotherapy. Int J Cancer 1999; 83:83-90. (4)Barber JBP et al. Relationship between in vitro chromosomal radiosensitivity of peripheral blood lymphocytes and the expression of normal tissue damage following radiotherapy for breast cancer. Radiother Oncol 2000; 55:179-186. (5)Scott D et al. Genetic predisposition in breast cancer. Lancet 1994;344:1444 (6) De Ruyck K et al. Chromosomal radiosensitivity in head and neck cancer patients: evidence for genetic predisposition ? Br J Cancer (in print) (7) De Ruyck K et al. Radiation-induced damage to normal tissues after radiotherapy in patients treated for gynaecological tumors: association with SNPs in XRCC1, XRCC3 and OGG1 and in vitro chromosomal radiosensitivity in lymphocytes. IJROBP 2005; 62:1140-1149. (8)De Ruyck K et al. TGF $\beta 1$ polymorphisms and late clinical radiosensitivity in patients treated for gynaecologic tumors. IJROBP 2006; 65:1240-1248. 